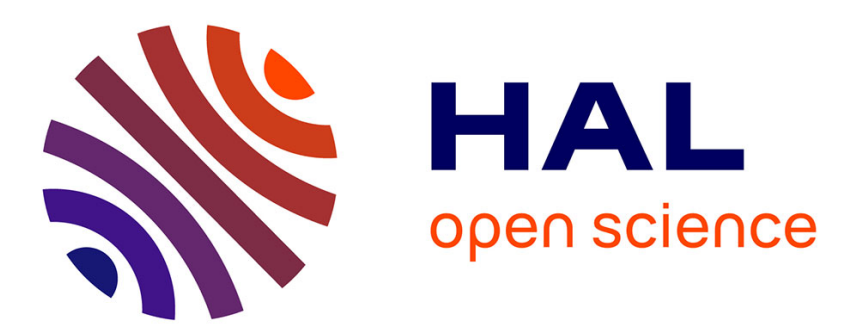

\title{
On integer-valued means and the symmetric maximum
}

\author{
Miguel Couceiro, Michel Grabisch
}

\section{To cite this version:}

Miguel Couceiro, Michel Grabisch. On integer-valued means and the symmetric maximum. Aequationes Mathematicae, 2017, 91 (2), pp.353-371. 10.1007/s00010-016-0460-9 . hal-01404593

\section{HAL Id: hal-01404593 \\ https://hal.inria.fr/hal-01404593}

Submitted on 29 Nov 2016

HAL is a multi-disciplinary open access archive for the deposit and dissemination of scientific research documents, whether they are published or not. The documents may come from teaching and research institutions in France or abroad, or from public or private research centers.
L'archive ouverte pluridisciplinaire HAL, est destinée au dépôt et à la diffusion de documents scientifiques de niveau recherche, publiés ou non, émanant des établissements d'enseignement et de recherche français ou étrangers, des laboratoires publics ou privés. 


\title{
On integer-valued means and the symmetric maximum
}

\author{
Miguel COUCEIRO ${ }^{1}$ and Michel GRABISCH${ }^{2}$ \\ 1. LORIA (CNRS - Inria Nancy Grand Est - Université de Lorraine), \\ Équipe Orpailleur, Batiment B, Campus Scientifique, B.P. 239, \\ F-54506 Vandoeuvre-lès-Nancy, France \\ 2. Paris School of Economics, University of Paris I \\ 106-112, Bd de l'Hôpital, 75013 Paris, France \\ Email: miguel.couceiro@inria.fr, michel.grabisch@univ-paris1.fr
}

September 21, 2016

\begin{abstract}
Integer-valued means, satisfying the decomposability condition of Kolmogoroff/Nagumo, are necessarily extremal, i.e., the mean value depends only on the minimal and maximal inputs. To overcome this severe limitation, we propose an infinite family of (weak) integer means based on the symmetric maximum and computation rules. For such means, their value depends not only on extremal inputs, but also on 2nd, 3rd, etc., extremal values as needed. In particular, we show that this family can be characterized by a weak version of decomposability.
\end{abstract}

Mathematics Subject Classification: 26E60, 08A99, 06A99

Keywords: integer means; nonassociative algebra; symmetric maximum; decomposability

\section{Introduction}

In 1930, Kolmogoroff [5] and Nagumo [7] independently characterized the class of quasiarithmetic means (including the well-known arithmetic, geometric and harmonic means) through a property which was later called decomposability [4], barycentric associativity [6], or simply associativity [1]. As associativity in the classical algebraic sense will play a central rôle in this paper, we prefer to adopt the term "decomposability".

Decomposability is a very natural property that amounts to saying that the average of a set of numbers should not be changed if a subset of it is replaced by its average. Denoting such an averaging function by $\mu$, the latter notion can be expressed by the condition

$$
\mu\left(a_{1}, \ldots, a_{n}\right)=\mu(\underbrace{b, \ldots, b}_{k \text { times }}, a_{k+1}, \ldots, a_{n})
$$

where $b=\mu\left(a_{1}, \ldots, a_{k}\right)$. 
More recently, Bennett et al. [1] investigated the effect of this property on integervalued averaging functions, that is, averaging functions defined and valued in $\mathbb{Z}$. This setting is both natural and relevant in practice since in many situations input values are integer, for instance, number of children, of items, etc., and in such cases mean values that are not integer bear no meaning. Surprisingly, by imposing decomposability in addition to the usual requirements of averaging functions (nondecreasingness, anonymity and internality), it turns out that such integer-valued averaging functions (called integer means or $\mathbb{Z}$-means in [1]) are extremal, that is, the output depends only on the minimum and maximum input values. In symbols,

$$
\mu\left(a_{1}, \ldots, a_{n}\right)=\mu\left(a_{1}, a_{n}\right)
$$

with $a_{1} \leq a_{2} \leq \cdots \leq a_{n}$. Put otherwise, integer means ignore all input values but the minimum and the maximum ones.

The aim of this paper is to attempt to finding a class of integer-valued averaging functions that have a less drastic behavior and whose output retains more information of the input, by allowing to considering not only minimal and maximal inputs, but also, when necessary, second, third, etc., $\min / \max$ values. This can be achieved through the use of the symmetric maximum, a symmetrization of the classical maximum operator proposed by Grabisch [3], and denoted by $\emptyset$. Roughly speaking, $\emptyset$ is a binary operator on $\mathbb{Z}$ yielding the extremal value of its inputs (i.e., the greatest in absolute value), except if these are opposite values, in which case the output is 0 , for instance

$$
a \otimes(-a)=0, \quad 2 \otimes 3=3, \quad(-2) \otimes 3=3, \quad 2 \otimes(-3)=-3 .
$$

As it is easy to see, such an operator is not associative in the algebraic sense. Indeed,

$$
(3 \otimes(-3)) \oslash 2=0 \otimes 2=2, \quad 3 \otimes((-3) \oslash 2)=3 \otimes(-3)=0 .
$$

Therefore, defining an averaging function by $\oslash$, i.e., writing $\oslash\left(a_{1}, \ldots, a_{n}\right)$ for any sequence $a_{1}, \ldots, a_{n}$ of inputs is meaningless without further refinement of the definition. In fact, nonassociativity arises if and only if there exist extremal opposite terms in the input sequence (like $3,-3)$, otherwise $\otimes\left(a_{1}, \ldots, a_{n}\right)$ is well defined. In order to overcome this drawback, Grabisch [3] proposed systematic ways to aggregate all terms of a sequence using a single binary operator as in (2). These systematic ways of putting parentheses were called rules of computation, and were fully investigated by the authors in [2]. A simple example of such a rule is the following: aggregate first all pairs of extremal opposite numbers in decreasing order, till no such pair exist. On the sequence of number $4,-4,3,-3,2,1$, this would yield:

$$
\oslash(4,-4,3,-3,2,1)=(4 \otimes(-4)) \otimes(3,-3,2,1)=0 \otimes(((3 \otimes(-3)) \otimes(2,1)=0 \otimes(2,1)=2 .
$$

(1) viewed as an integer-valued averaging function is clearly not extremal, but as said above, it satisfies extremality in a wider sense: in the above example we have

$$
\oslash(4,-4,3,-3,2,1)=\emptyset(2,1)
$$

and $(2,1)$ is the $3 \mathrm{~d}$ pair of $\min / \max$ inputs, the two first pairs having been eliminated because they are pairs of opposite values. 
As shown in [2], infinitely many rules of computation exist, yielding an infinite (and even uncountable) family of integer-valued averaging functions. Interestingly, none of them satisfy decomposability (Corollary 1), but they satisfy a weaker version of it, hence we call them weak integer means. More precisely, the decomposability property (1) is valid only for certain subsequences of $a_{1}, \ldots, a_{n}$ (Lemma 2).

We provide a complete characterization of this family of weak integer means, by using weak decomposability (Theorem 3), or a related property called the replacement property (Lemma 5). The various characterizations are summarized in Theorem 4.

\section{Preliminaries}

\section{$2.1 \mathbb{Z}$-means and the symmetric maximum}

We consider a countable set $C$ which is totally ordered by $\leqslant$, and with minimal element 0. Let $C^{-}=\{-c \mid c \in C\}$ be its dually isomorphic copy, and consider $\tilde{C}=C \cup C^{-}$, setting $-0=0 .{ }^{1}$ For convenience, we introduce the notation

$$
\tilde{C}^{*}=\left(\bigcup_{n \in \mathbb{N}}(\tilde{C})^{n}\right) \cup\{\varepsilon\},
$$

where $\varepsilon$ is the empty string. Put differently, $\tilde{C}^{*}$ is the set of all words built over the alphabet $\tilde{C}$ using concatenation.

Throughout the paper we deal with functions $\mu: \tilde{C}^{*} \rightarrow \tilde{C}$, and always adopt the following convention: $\mu(\varepsilon)=0$ and $\mu(a)=a$ for all $a \in \tilde{C}$.

We recall the main result from [1]. A function

$$
\mu: \tilde{C}^{*} \rightarrow \tilde{C}
$$

is a $\mathbb{Z}$-mean or integer-valued mean if it satisfies the following properties:

(i) Anonymity: for every $n \in \mathbb{N}$, every permutation $\pi$ on $[n]$,

$$
\mu\left(a_{1}, \ldots, a_{n}\right)=\mu\left(a_{\pi(1)}, \ldots, a_{\pi(n)}\right) ;
$$

(ii) Internality: for every $n \in \mathbb{N}$, if $a_{1} \leqslant \cdots \leqslant a_{n}$,

$$
a_{1} \leqslant \mu\left(a_{1}, \ldots, a_{n}\right) \leqslant a_{n}
$$

(iii) Monotonicity: for every $n \in \mathbb{N}, a_{i} \leqslant a_{i}^{\prime}$ for $i=1, \ldots, n$ implies

$$
\mu\left(a_{1}, \ldots, a_{n}\right) \leqslant \mu\left(a_{1}^{\prime}, \ldots, a_{n}^{\prime}\right) ;
$$

\footnotetext{
${ }^{1} \mathrm{~A}$ typical example is $\tilde{C}=\mathbb{Z}$, and in fact any such $\tilde{C}$ is isomorphic to $\mathbb{Z}$ or to a subset of it. This is why we may call elements of $\tilde{C}$ "integers" and use the corresponding terminology, like positive and negative numbers, absolute value, etc.
} 
(iv) Decomposability ${ }^{2}$ : for every $k, n \in \mathbb{N}$ with $k \leq n$ and $\mu\left(a_{1}, \ldots, a_{k}\right)=b$, we have

$$
\mu\left(a_{1}, \ldots, a_{k}, a_{k+1}, \ldots, a_{n}\right)=\mu(\underbrace{b, \ldots, b}_{k}, a_{k+1}, \ldots, a_{n}) .
$$

A function $\mu: \tilde{C}^{*} \rightarrow \tilde{C}$ is extremal if for any $n \in \mathbb{N}$ and any $a_{1} \leq \cdots \leq a_{n}$, we have

$$
\mu\left(a_{1}, \ldots, a_{n}\right)=\mu\left(a_{1}, a_{n}\right)
$$

Theorem 1. All $\mathbb{Z}$-means are extremal.

Hence, $\mathbb{Z}$-means, unlike means defined on real numbers, do not consider all values to be aggregated but only the extreme ones. Our aim is to overcome this rather negative result by weakening the definition of decomposability, which is mainly responsible for extremality, and to propose a whole family of aggregation functions fulfilling decomposability in a weak sense. This will be achieved through the symmetric maximum [3], which is a binary operation $\emptyset: \tilde{C}^{2} \rightarrow \tilde{C}$ defined by

$$
a \otimes b= \begin{cases}-(|a| \vee|b|), & \text { if } b \neq-a \text { and }|a| \vee|b|=-a \text { or }-b \\ 0, & \text { if } b=-a \\ |a| \vee|b|, & \text { otherwise. }\end{cases}
$$

In other words, except if $a=-b, a \bigotimes b$ equals the element among $a, b$ that is extremal, i.e., that has the largest absolute value (magnitude). It is not difficult to see that $\oslash$ satisfies the following properties:

(i) (C1) $\oslash$ coincides with the maximum on $C^{2}$;

(ii) $(\mathrm{C} 2) a \otimes(-a)=0$ for every $a \in \tilde{C}$;

(iii) $(\mathrm{C} 3)-(a \otimes b)=(-a) \otimes(-b)$ for every $a, b \in \tilde{C}$.

By $(\mathrm{C} 1),(\mathrm{C} 3), \otimes$ coincides with the minimum on $\left(C^{-}\right)^{2}$. Also, it is monotone, internal, commutative (or symmetric), and 0 is its neutral element (by $(\mathrm{C} 1)$ and $(\mathrm{C} 3)$ ). Hence, $\mathbb{(}$ almost behaves like a commutative group operation on $\tilde{C}$, however it lacks associativity: it is not true in general that

$$
a \oslash(b \otimes c)=(a \oslash b) \otimes c
$$

as shown by the following example:

$$
(-3 \otimes 3) \otimes 1=0 \otimes 1=1 ; \quad-3 \otimes(3 \otimes 1)=-3 \otimes 3=0 .
$$

The following result gives an algebraic characterization of $\otimes$ and shows that if one requires that (C1), (C2) and (C3) hold, then (3) is the best possible definition for $\oslash$.

Theorem 2. [3, Prop. 5] No binary operation satisfying (C1), (C2), (C3) fulfills (4) on a larger domain than $\varnothing$.

\footnotetext{
${ }^{2}$ This property is called associativity in [1], and barycentric associativity in [6]. However, the usual meaning of associativity in algebra is different, and the latter is a central property in this paper. To avoid any confusion, we therefore stick to the term "decomposability".
} 
It is easy to see that the domain where $\oslash$ is associative is given by the set of triples $(a, b, c)$ such that

$$
\min (a, b, c) \neq-\max (a, b, c) .
$$

Thorem 2 states that under $(\mathrm{C} 1),(\mathrm{C} 2)$ and (C3), any binary operation $\mu$ on $\tilde{C}$ is nonassociative as soon as (5) is not satisfied (i.e., for triples with opposite extremal elements). Moreover, if $\mu \neq \emptyset$, then there exists a triple satisfying (5) for which $\mu$ is nonassociative. For instance, $\stackrel{0}{\vee}$ defined by

$$
\stackrel{0}{\vee}\left(a_{1}, \ldots, a_{n}\right)= \begin{cases}a_{1}, & \text { if } a_{n} \leqslant 0 \\ 0, & \text { if } a_{1}<0<a_{n} \\ a_{n}, & \text { if } a_{1} \geqslant 0\end{cases}
$$

with $a_{1} \leqslant \cdots \leqslant a_{n}$, satisfies (C1), (C2) and (C3), but

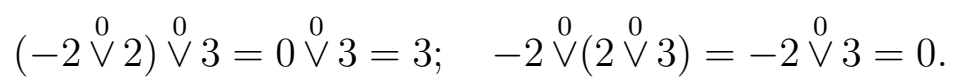

\subsection{How to make $\otimes$ associative: Rules of computation}

The lack of associativity implies that it is not possible to extend the definition of $\oslash$ to $\tilde{C}^{*}$, since the result depends on the particular way that we apply the binary $\emptyset$ to the terms of a sequence with more than two terms. Therefore, no function like a $\mathbb{Z}$-mean can be defined based on this operation. However, a way to overcome this difficulty, suggested in [3] and fully developed in [2], is to fix beforehand a systematic way of putting parentheses on any sequence of $\tilde{C}^{*}$, procedure that was called a rule of computation.

Elements of $\tilde{C}^{*}$ are called sequences (of "integers"), denoted by $\sigma=\left(a_{i}\right)_{i \in I}$ for some finite index set $I$, and $a_{i} \in \tilde{C}$ for all $i \in I$ (note that 0 is a possible value, contrarily to $[2])$. Since we consider only anonymous functions on $\tilde{C}^{*}$, the order is unimportant and so we consider only sequences of integers in decreasing order of their absolute value, positive integers first (e.g., $5,5,-5,-3,2,-2,1,0)$. The empty sequence is $\varepsilon$. The set of all such integer sequences, including the empty sequence, is denoted by $\mathfrak{S}$. Since sequences are ordered as described above, a convenient notation is the following. For an arbitrary sequence

$$
\sigma=(\underbrace{n_{1}, \ldots, n_{1}}_{p_{1} \text { times }}, \underbrace{-n_{1}, \ldots,-n_{1}}_{m_{1} \text { times }}, \ldots, \underbrace{n_{q}, \ldots, n_{q}}_{p_{q} \text { times }}, \underbrace{-n_{q}, \ldots,-n_{q}}_{m_{q} \text { times }})
$$

we introduce $\theta(\sigma)=\left(n_{1}, \ldots, n_{q}\right)$, the sequence of absolute values (magnitudes) of integers in $\sigma$, and $\psi(\sigma)=\left(\left(p_{1}, m_{1}\right), \ldots,\left(p_{q}, m_{q}\right)\right)$ all numbers of occurrence of these integers.

Example 1. Consider the sequence $\sigma=(3,3,-3,2,-2,-2,1,1,1,1)$. Then

$$
\theta(\sigma)=(3,2,1) ; \quad \psi(\sigma)=((2,1),(1,2)(4,0)) .
$$

A sequence $\left(a_{i}\right)_{i \in I}$ fulfills associativity (or, simply, is associative) if $\min _{i \in I} a_{i} \neq-\max _{i \in I} a_{i}$ (equivalently, if either $p_{1}=0$ or $m_{1}=0$ ). The set of sequences which do not fulfill associativity is denoted by $\mathfrak{S}_{0}$. Note that by this definition the sequence $(1,-1)$, although containing only 2 elements, is not associative.

The above notation permits to introduce rules of computation in a proper way. We define 5 elementary rules $\rho_{i}: \mathfrak{S} \rightarrow \mathfrak{S}$ which operate on $\sigma$ as follows: 
(i) Elementary rule $\rho_{1}$ : if $p_{1}>1$ and $m_{1}>0$, then $p_{1}$ is changed to $p_{1}=1$;

(ii) Elementary rule $\rho_{2}$ : idem with $p_{1}, m_{1}$ exchanged;

(iii) Elementary rule $\rho_{3}$ : if $p_{1}>0, m_{1}>0$, the pair $\left(p_{1}, m_{1}\right)$ is changed into $\left(p_{1}-c, m_{1}-\right.$ $c)$, where $c=p_{1} \wedge m_{1}$;

(iv) Elementary rule $\rho_{4}$ : if $p_{1}>0, m_{1}>0$, and if $p_{2}>0$, then $p_{2}$ is changed into $p_{2}=0$;

(v) Elementary rule $\rho_{5}$ : idem with $m_{2}$ replacing $p_{2}$.

Hence, elementary rules delete terms in sequences which do not fulfill associativity, and do nothing otherwise.

A (well-formed) computation rule $R$ is a word built with the alphabet $\left\{\rho_{1}, \ldots, \rho_{5}\right\}$, i.e., $R \in \mathcal{L}\left(\rho_{1}, \ldots, \rho_{5}\right)$, such that $R(\sigma) \in \mathfrak{S} \backslash \mathfrak{S}_{0}$ for all $\sigma \in \mathfrak{S}$. The set of (well-formed) computation rules is denoted by $\mathfrak{R}$. Examples of rules are (words are read from left to right)

(i) $\langle\cdot\rangle_{0}=\rho_{3}^{*}$;

(ii) $\langle\cdot\rangle_{=}=\left(\rho_{1} \rho_{2} \rho_{3}\right)^{*}$;

(iii) $\langle\cdot\rangle_{-}^{+}=\left(\rho_{4} \rho_{5}\right)^{*} \rho_{1} \rho_{2} \rho_{3}$.

We use the notation $\emptyset_{R}$ for $\oslash \circ R$. It is shown in [2] that any computation rule has the form $R=T_{1} T_{2} \cdots$, where each $T_{i}$ has the form $\omega \rho_{1}^{\alpha} \rho_{2}^{\beta} \rho_{3}$, with $\omega \in \mathcal{L}\left(\rho_{4}, \rho_{5}\right)$ and $\alpha, \beta \in\{0,1\}$ (factorization scheme). ${ }^{3}$ Moreover, $\mathfrak{R}$ is uncountable (the set of its maximal elements is isomorphic to $\left.2^{\mathbb{N}}\right)$. We denote by $\mathfrak{R}_{123}$ the set of rules where $\omega=\varepsilon$ in each term $T_{i}$ (i.e., the rule uses only $\left.\rho_{1}, \rho_{2}, \rho_{3}\right)$. Also, we introduce $\operatorname{Ker}(R)=\{\sigma \in \mathfrak{S} \mid R(\sigma)=\varepsilon\}$, the kernel of $R$.

Two rules $R, R^{\prime}$ are equivalent (denoted by $R \sim R^{\prime}$ ) if $\bigotimes_{R}=\bigotimes_{R^{\prime}}$ on $\mathfrak{S}$. For a given rule $R$ and a sequence $\sigma=\left(a_{i}\right)_{i \in I}$, we denote by $J_{\sigma}^{R}$ the set of elements deleted by $R$ in $\sigma$. We use the following notation: for a sequence $\left(a_{i}\right)_{i \in I}$ and any set $K \subseteq I$, we write $a_{K}$ for the subsequence $\left(a_{i}\right)_{i \in K}$. Hence, we may write

$$
\left(a_{i}\right)_{i \in I}=\left(a_{K}, a_{I \backslash K}\right) .
$$

For convenience, we may also write $\sigma_{K}$ for a restriction of the sequence $\sigma$ to $K$.

\subsection{The basic mechanism and properties of $\otimes_{R}$}

It is important to analyse the effect of a rule $R=T_{1} T_{2} \cdots$ (not necessarily in $\mathfrak{R}$ ) on a sequence $\sigma \in \mathfrak{S}_{0}$, with representation

$$
\psi(\sigma)=\left(\left(p_{1}, m_{1}\right), \ldots,\left(p_{r}, m_{r}\right)\right), \quad \theta(\sigma)=\left(n_{1}, \ldots, n_{r}\right) .
$$

Let us first consider a single term $T_{1}=\omega \rho$, where $\rho$ is a shorthand for $\rho_{1}^{\alpha} \rho_{2}^{\beta} \rho_{3}$. It is straightforward to verify that $T_{1}(\sigma)$ is another sequence with representation

$$
\psi\left(T_{1}(\sigma)\right)=\left(\left(p_{1}^{\prime}, m_{1}^{\prime}\right),\left(p_{q}^{\prime}, m_{q}^{\prime}\right),\left(p_{q+1}, m_{q+1}\right), \ldots,\left(p_{r}, m_{r}\right)\right), \quad \theta\left(T_{1}(\sigma)\right)=\left(n_{1}, n_{q}, \ldots, n_{r}\right)
$$

\footnotetext{
${ }^{3}$ Here, we adopt the notation $\rho^{0}=\varepsilon$ and $\rho^{1}=\rho$.
} 
with the following requirements:

$$
\begin{aligned}
& 0 \leqslant p_{1}^{\prime}<p_{1}, \quad 0 \leqslant m_{1}^{\prime}<m_{1}, \text { and at most one of them is positive } \\
& 2 \leqslant q \leqslant r \\
& p_{q}^{\prime}=0 \text { or } p_{q}, \quad m_{q}^{\prime}=0 \text { or } m_{q}
\end{aligned}
$$

This situation is pictured in Figure 1. The first hatched block is the part deleted by

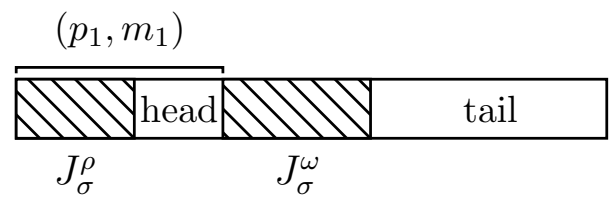

Figure 1: The sequences $\sigma$ and $T_{1}(\sigma)$ (white part), with values from left to right in decreasing order of their magnitude. The hatched part is $J_{\sigma}^{T_{1}}$

$\rho$, while the second one is deleted by $\omega$. The "head" is the undeleted part of $\left(p_{1}, m_{1}\right)$ between the two hatched blocks and it may be the case that it does not exist. It contains only $n_{1}$ or only $-n_{1}$, with possible repetitions. The "tail" is the part left undeleted by $\omega$ and may not exist. The left hatched block always exist, while the second may not, in which case we say that the head is empty.

Applying successively the different terms $T_{2}, T_{3}, \ldots$ of $R$ it is not difficult to see that the final result $R(\sigma)$ has almost the same form. Indeed, if a head exists in $T_{1}(\sigma)$, then $T_{1}(\sigma)$ is associative, and the rule stops. If not, the next term applies (if nonassociative), yielding the same form as above. Hence the representation for $R(\sigma)$ is

$$
\psi(R(\sigma))=\left(\left(p_{k}^{\prime}, m_{k}^{\prime}\right),\left(p_{q}^{\prime}, m_{q}^{\prime}\right),\left(p_{q+1}, m_{q+1}\right), \ldots,\left(p_{r}, m_{r}\right)\right), \quad \theta(R(\sigma))=\left(n_{k}, n_{q}, \ldots, n_{r}\right)
$$

with the following requirements:

$$
\begin{aligned}
& k \geqslant 1 \\
& 0 \leqslant p_{k}^{\prime} \leqslant p_{k}, \quad 0 \leqslant m_{k}^{\prime} \leqslant m_{k}, \text { and at most one of them is positive } \\
& k+1 \leqslant q \leqslant r \\
& p_{q}^{\prime}=0 \text { or } p_{q}, \quad m_{q}^{\prime}=0 \text { or } m_{q}
\end{aligned}
$$

This situation is pictured in Figure 2. We define the head and tail of $R(\sigma)$ similarly:

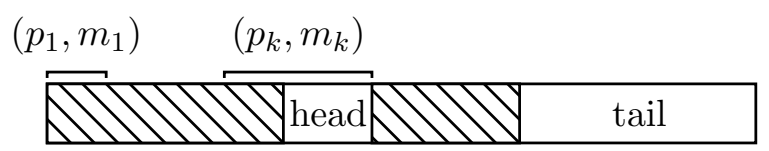

Figure 2: The sequences $\sigma$ and $R(\sigma)$ (white part), with values from left to right in decreasing order of their magnitude. The hatched part is $J_{\sigma}^{R}$

the head is the part left undeleted by $R$ between the two deleted parts, while the tail is 
the part left undeleted which has no deleted part on its right side. If the head exists, it contains only value $n_{k}$ or only $-n_{k}$, possibly with repetition. Note that when they both exist, the elements in the tail have strictly smaller magnitude than any element of the head.

We denote by $H, T$ the subsets of $I$ corresponding to the head and tail of a sequence $\sigma=\left(a_{i}\right)_{i \in I}$, and therefore may use the notation $\sigma_{H}, \sigma_{T}$ or $a_{H}, a_{T}$ for the head and tail.

Based on this result, we make some useful observations.

Lemma 1. Let $\sigma=\left(a_{i}\right)_{i \in I} \in \mathfrak{S}_{0}$. The following holds.

(i) $\bigotimes_{R}(\sigma)=\bigotimes_{R}\left(a_{I \backslash J_{\sigma}^{R}}\right)=\otimes\left(a_{I \backslash J_{\sigma}^{R}}\right)=\left(\min _{i \in I \backslash J_{\sigma}^{R}} a_{i}\right) \otimes\left(\max _{i \in I \backslash J_{\sigma}^{R}} a_{i}\right)$ for any $R \in \mathfrak{R}$;

(ii) $\bigotimes_{R}$ is odd for any $R \in \Re$, i.e., $\bigotimes_{R}(-\sigma)=-\bigotimes_{R}(\sigma)$;

(iii) Let $R=T_{1} T_{2} \cdots$. If $R(\sigma)$ has a (nonempty) head, then $\bigotimes_{R}\left(\sigma_{I \backslash T}, \sigma_{T}\right)=\bigotimes_{R}\left(\sigma_{I \backslash T}, \sigma^{\prime}\right)$, where $\sigma^{\prime}$ is any sequence (possibly empty); If $R(\sigma)$ has no head, then $\bigotimes_{R}\left(\sigma_{I \backslash T}, \sigma_{T}\right)=$ $\bigotimes_{R}\left(\sigma_{I \backslash T}, \sigma^{\prime}\right)$, for any $\sigma^{\prime} \in \mathfrak{S} \backslash \mathfrak{S}_{0}$ which has the same extremal value as $\sigma_{T}$;

(iv) $a_{J_{\sigma}^{R}} \in \operatorname{Ker}(R)$, hence $\bigotimes_{R}\left(a_{J_{\sigma}^{R}}\right)=0$, for any $R$ of the form $T_{1} T_{2} \cdots$ (finite or infinite);

(v) For any $R \in \mathfrak{R}$, letting $\bigotimes_{R}(\sigma)=b$, then $|b| \leqslant \max _{i \in J_{\sigma}^{R}}\left|a_{i}\right|$, and $|b| \geqslant\left|a_{i}\right|$ for all $i \in I \backslash J_{\sigma}^{R}$ (and there is equality for some $i$, if $I \backslash J_{\sigma}^{R} \neq \varnothing$ );

Proof. (i) The first equality comes from $\bigotimes_{R}(\sigma)=\bigotimes(R(\sigma))=\emptyset\left(a_{I \backslash J_{\sigma}^{R}}\right)$. By definition of a computation rule, $a_{I \backslash J_{\sigma}^{R}}$ fulfills associativity, which explains the second equality, while the third one follows from associativity and the definition of $\emptyset$.

(ii) From (i) and (C3).

(iii) Suppose $R(\sigma)$ has a head. Then $\oslash_{R}(\sigma)=b$ is the value in the head, which is strictly greater in magnitude than any value in the tail. Therefore the tail has no effect on the result. Otherwise, $b$ is the extremal value of the tail. Hence the tail can be modified provided that it remains associative and with same extremal value.

(iv) Step 1: We consider first that $R=T_{1}=\omega \rho_{1}^{\alpha} \rho_{2}^{\beta} \rho_{3}$ and we denote $\rho=\rho_{1}^{\alpha} \rho_{2}^{\beta} \rho_{3}$ for short. Consider a sequence $\sigma=\left(a_{i}\right)_{i \in I}$ and let $\psi(\sigma)=\left(\left(p_{1}, m_{1}\right), \ldots,\left(p_{k}, m_{k}\right)\right), \psi\left(a_{J_{\sigma}^{R}}\right)=$ $\left(\left(p_{1}^{\prime}, m_{1}^{\prime}\right), \ldots,\left(p_{k^{\prime}}^{\prime}, m_{k^{\prime}}^{\prime}\right)\right)$. Observe that $p_{1}^{\prime}>0, m_{1}^{\prime}>0$ since $\rho_{3}$ is present in $T_{1}$. Therefore, if $\varepsilon \neq \omega=\omega_{1} \omega_{2} \cdots, \omega_{1}$ applies, then the remaining $\omega_{2}$, etc., also apply because no $\omega_{i}$ modifies $p_{1}^{\prime}, m_{1}^{\prime}$. It then follows that $J_{\sigma}^{\omega}=J_{a_{J_{\sigma}^{R}}}^{\omega}$. Now, observe that $\rho$ modifies only $\left(p_{1}, m_{1}\right)$, hence $J_{\omega(\sigma)}^{\rho}=J_{\omega\left(a_{J_{\sigma}^{R}}\right)}^{\rho}$, and thus $R\left(\sigma_{J_{\sigma}^{R}}\right)=\varepsilon$.

Step 2: We consider now the case where $R$ is not limited to a single term $T_{1}$ and we show the result by induction on the size of the sequence.

Induction hypothesis: for any $\sigma=\left(a_{i}\right)_{i \in I} \in \mathfrak{S}_{0},|\sigma| \leqslant t$ with $t \geqslant 3$, for any $R=$ $T_{1} T_{2} \cdots, R\left(a_{J_{\sigma}^{R}}\right)=\varepsilon$.

Step 2.1: We show the base case with $t=3$. Any sequence in $\mathfrak{S}_{0}$ of three terms is of the form $(a,-a, b)$ with $|a| \geqslant|b|$. Then only $T_{1}$ is applied in $R$, and it is easy to check that the result holds in any case.

Step 2.2: We now consider $\sigma=\left(a_{i}\right)_{i \in I}$ in $\mathfrak{S}_{0}$ with $|\sigma|=t+1$ and representation $\psi(\sigma)=\left(\left(p_{1}, m_{1}\right), \ldots,\left(p_{q}, m_{q}\right)\right), q \geqslant 1$. Let $T_{1}=\omega \rho_{1}^{\alpha} \rho_{2}^{\beta} \rho_{3}$ and consider the rule $R^{\prime}=$ $T_{2} T_{3} \cdots$ (which may have only one term, in which case Step 1 of the proof must be 
used). Observe that since $p_{1}>0$ and $m_{1}>0, \omega$ does not modify $p_{1}, m_{1}$ and $\rho_{1}, \rho_{2}$ leave $p_{1}>0, m_{1}>0$, the action of $\rho_{3}$ will decrease $p_{1}$ and $m_{1}$ by at least one unit each. It then follows that $\left|T_{1}(\sigma)\right| \leqslant|\sigma|-2=t-1$.

Moreover, by definition we have $R(\sigma)=R^{\prime}\left(T_{1}(\sigma)\right)$ and so $J_{\sigma}^{R}=J_{\sigma}^{T_{1}} \cup J_{T_{1}(\sigma)}^{R^{\prime}}$. Applying induction hypothesis, we find

$$
\varepsilon=R^{\prime}\left(\left(T_{1}(\sigma)\right)_{J_{T_{1}(\sigma)}^{R^{\prime}}}\right)
$$

We claim that $R^{\prime}\left(\left(T_{1}(\sigma)\right)_{J_{T_{1}(\sigma)}^{R^{\prime}}}\right)=R\left(\sigma_{J_{\sigma}^{R}}\right)$, which finishes the proof.

Proof of the Claim: We have by definition

$$
R\left(\sigma_{J_{\sigma}^{R}}\right)=R^{\prime}\left(T_{1}\left(\sigma_{J_{\sigma}^{R}}\right)\right)=R^{\prime}\left(T_{1}\left(\sigma_{J_{\sigma}^{T_{1}}}, \sigma_{J_{T_{1}(\sigma)}^{R^{\prime}}}\right)\right)
$$

It remains then to prove

$$
T_{1}\left(\sigma_{J_{\sigma}^{T_{1}}}, \sigma_{J_{T_{1}(\sigma)}^{R^{\prime}}}\right)=\left(T_{1}(\sigma)\right)_{J_{T_{1}(\sigma)}^{R^{\prime}}} .
$$

We distinguish several cases on the structure of $R(\sigma)$, distinguishing the effect of $T_{1}$ and $R^{\prime}$. In the following figures, the hatched and the grey parts are respectively the effect of $T_{1}$ and $R^{\prime}$. The second hatched block may be empty (if $\omega=\varepsilon$ ), and similarly for the second grey block. We have to show that applying $T_{1}$ to the sequence formed by the hatched and grey parts (called $\sigma^{\prime}$ ) deletes exactly the hatched part. The foregoing analysis shows that only three cases can happen.

- Case 1:

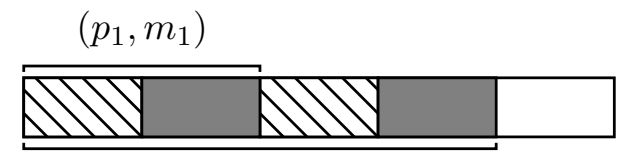

$$
\sigma^{\prime}
$$

Observe that the action of $\omega$ is on the second hatched part, and depends only on $\left(p_{1}, m_{1}\right)$ and the current $\left(p_{k}, m_{k}\right)$ and not on subsequent values. As these pairs are unchanged, the action of $\omega$ on $\sigma^{\prime}$ or on $\sigma$ is exactly the same. Now, $\rho$ acts only on $\left(p_{1}, m_{1}\right)$ and depends only on $\left(p_{1}, m_{1}\right)$. Hence, the result on $\sigma$ and $\sigma^{\prime}$ is again the same.

- Case 2:

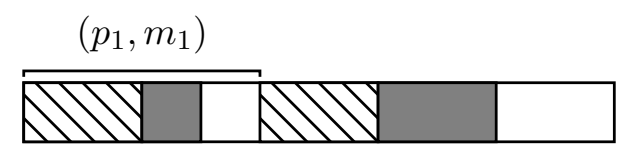

Here $\left(p_{1}, m_{1}\right)$ is changed into $\left(p_{1}^{\prime}, m_{1}^{\prime}\right)$ in $\sigma^{\prime}$, but both $p_{1}^{\prime}, m_{1}^{\prime}$ are positive due to the presence of $\rho_{3}$ in $T_{1}$. Hence the same reasoning as in case 1 applies.

$$
\left(p_{1}, m_{1}\right)
$$

- Case 3:

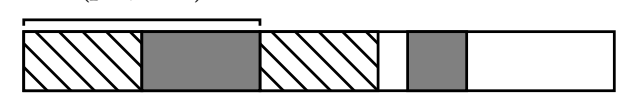

This case is treated like case 1 .

(v) Clear from the foregoing analysis with head and tail. 


\section{Weak $\mathbb{Z}$-means based on the symmetric maximum}

Specifically, we say that $\mu: \tilde{C}^{*} \rightarrow \tilde{C}$ is a weak $\mathbb{Z}$-mean if it satisfies anonymity, internality, monotonicity and restricted decomposability: for every sequence $\left(a_{i}\right)_{i \in I}$, there exists at least one nonempty $K \subseteq I$ on which decomposability applies:

$$
\mu\left(a_{K}\right)=b \text { implies } \mu\left(a_{K}, a_{I \backslash K}\right)=\mu\left(|K| \cdot b, a_{I \backslash K}\right),
$$

where $k \cdot b$, for some nonnegative integer $k$ and some $b \in \tilde{C}$, means $b, b, \ldots, b$ (repeated $k$ times). In this case we say that $\mu$ is decomposable for $K$.

We recall that an anonymous function $\mu: \tilde{C}^{*} \rightarrow \tilde{C}$ is associative if $\mu(a)=a$ for every $a \in \tilde{C}$, and for every sequence $\sigma=\left(a_{i}\right)_{i \in I}$ and $K \subset I$, we have $\mu(\sigma)=\mu\left(\mu\left(a_{K}\right), a_{I \backslash K}\right.$ ) (see $[4, \S 2.3 .1])$. We say that an anonymous function $\mu$ is weakly associative if associativity holds for associative sequences (i.e., on $\mathfrak{S} \backslash \mathfrak{S}_{0}$ ).

We show in this section that the symmetric maximum is the basis for an infinite (uncountable) family of weakly associative weak $\mathbb{Z}$-means, namely $\left(\mathbb{Q}_{R}\right)_{R \in \mathfrak{R}}$.

Lemma 2. Let $R \in \mathfrak{R}$.

(i) For every sequence $\sigma=\left(a_{i}\right)_{i \in I}, \bigotimes_{R}$ satisfies decomposability for every $K \supseteq J_{\sigma}^{R}$ and for every nonempty $K^{\prime} \subseteq I \backslash J_{\sigma}^{R}$;

(ii) For each rule $R$, there exists a sequence $\sigma=\left(a_{i}\right)_{i \in I}$ in $\mathfrak{S}_{0}$ and a set $K \subseteq I$ such that $\otimes_{R}$ is not decomposable for $K$.

Proof. (i) We know by Lemma 1 (iv) that $\otimes_{R}\left(a_{J_{\sigma}^{R}}\right)=0$. Then, by Lemma 1 (i) and the fact that 0 is neutral,

$$
\bigotimes_{R}\left(\left|J_{\sigma}^{R}\right| \cdot 0, a_{I \backslash J_{\sigma}^{R}}\right)=\bigotimes_{R}\left(a_{I \backslash J_{\sigma}^{R}}\right)=\bigotimes(\sigma),
$$

hence decomposability holds for $J_{\sigma}^{R}$.

Let $K \supset J_{\sigma}^{R}$ and let $b^{\prime}=\bigotimes_{R}\left(a_{K}\right)$. We have to prove that

$$
\oslash_{R}\left(|K| \cdot b^{\prime}, a_{I \backslash K}\right)=\oslash_{R}(\sigma)=: b .
$$

Suppose that $R(\sigma)$ has a head, and hence $b$ is in the head. Then $J_{a_{K}}^{R}=J_{\sigma}^{R}$ and $\left|b^{\prime}\right| \leqslant|b|$. Moreover $b^{\prime}=b$ holds iff $K$ intersects the head of $\sigma$, otherwise strict inequality occurs and $b^{\prime}$ is the extremal value of the tail. Then $\left(|K| \cdot b^{\prime}, a_{I \backslash K}\right)$ is associative in any case, because the head does not contain $-b$ and the tail is associative. Its extremal value is $b$ in any case, because either $K$ contains the head, and then $b^{\prime}=b$, otherwise it does not and $b$ is present in $a_{I \backslash K}$.

Suppose now that $R(\sigma)$ has no head. Observe that the sequence restricted to $K \cap I \backslash J_{\sigma}^{R}$ is not necessarily associative, therefore $J_{a_{K}}^{R} \supseteq J_{\sigma}^{R}$, but anyway $\left|b^{\prime}\right| \leqslant|b|$. If $\left|b^{\prime}\right|=|b|$, then the sequence restricted to $K \cap I \backslash J_{\sigma}^{R}$ is associative and $b^{\prime}=b$ because no $-b$ is present in the tail. Then $J_{a_{K}}^{R}=J_{\sigma}^{R}$ and $\oslash_{R}\left(|K| \cdot b, a_{I \backslash K}\right)=b$. Suppose on the contrary that $\left|b^{\prime}\right|<|b|$. Then the sequence $\left(|K| \cdot b^{\prime}, a_{I \backslash K}\right)$ is associative because $b$ is extremal and no $-b$ is present in the tail, therefore $\bigotimes_{R}\left(|K| \cdot b^{\prime}, a_{I \backslash K}\right)=b$.

Consider finally $K^{\prime} \subseteq I \backslash J_{\sigma}^{R}$ and let $b^{\prime}=\bigotimes_{R}\left(a_{K^{\prime}}\right)$. 
Suppose that $R(\sigma)$ has a head, and that $K$ intersects the head. Then clearly $b^{\prime}=b$ and $\otimes_{R}\left(\left|K^{\prime}\right| \cdot b, a_{I \backslash K^{\prime}}\right)=b$, as desired. If on the contrary $K^{\prime}$ is included in the tail, then $\left|b^{\prime}\right|<|b|$. By Lemma 1(iii), $\oslash_{R}\left(\left|K^{\prime}\right| \cdot b^{\prime}, a_{I \backslash K^{\prime}}\right)=b$.

Suppose now that $R(\sigma)$ has no head, which implies that $K^{\prime}$ is included in the tail. Then $\left|b^{\prime}\right| \leqslant|b|$. If equality occurs, then $b^{\prime}=b, a_{K^{\prime}}$ is associative and the modified tail remains associative. It follows that the desired result holds by Lemma 1(iii). If $\left|b^{\prime}\right|<|b|$, the tail remains also associative because there is no $-b$. Again, the result holds by Lemma 1(iii).

(ii) We prove now that for each rule, one can find a nonassociative sequence and a subsequence of it on which the symmetric maximum is not decomposable. Consider the sequence $\sigma=(2,-2,1)$. Suppose $R \in \mathfrak{R}_{123}$ and take the subsequence $\sigma_{K}=(-2,1)$. Then $\oslash_{R}(-2,1)=-2$ and $\oslash_{R}(\sigma)=1$. However, $\bigotimes_{R}(2,-2,-2)$ is equal to either 0 or -2 , depending whether $R$ uses $\rho_{2}$ or not. Hence decomposability fails for $K$.

Suppose now that $R \notin \Re_{123}$ and consider the subsequence $\sigma_{K}=(2,-2)$. Then $\oslash_{R}(2,-2)=0$ and $\oslash_{R}(\sigma)=0$ (supposing that $R$ contains $\rho_{4}$. Otherwise, replace $\sigma$ by $(2,-2,-1))$. However, $\bigotimes_{R}(0,0,1)=1$, and decomposability again fails on $K$.

Corollary 1. (i) The symmetric maximum is decomposable for no computation rule.

(ii) The symmetric maximum is decomposable for any sequence in $\mathfrak{S} \backslash \mathfrak{S}_{0}$, for any computation rule.

Observe also that $\langle\cdot\rangle_{-}^{+}$is the only rule yielding an extremal operator. Indeed,

$$
\oslash\left(\left\langle\left(a_{i}\right)_{i \in I}\right\rangle_{-}^{+}\right)=\left(\min _{i \in I} a_{i}\right) \otimes\left(\max _{i \in I} a_{i}\right) .
$$

Any other rule (as shown in [2]) deletes less terms than $\langle\cdot\rangle_{-}^{+}$, hence there are sequences for which other elements than the minimum and maximum are used. This fact motivates the definition of a weaker notion of extremality (see below).

For characterization purposes, we introduce a number of properties.

Definition 1. Let $\mu: \tilde{C}^{*} \rightarrow \tilde{C}$.

(i) $\mu$ is extremal w.r.t. $R \in \mathfrak{R}$ if for every $\sigma=\left(a_{i}\right)_{i \in I}$ in $\mathfrak{S}$

$$
\mu(\sigma)=\left(\min _{i \in I \backslash J_{\sigma}^{R}} a_{i}\right) \otimes\left(\max _{i \in I \backslash J_{\sigma}^{R}} a_{i}\right) ;
$$

(ii) $\mu$ satisfies the replacement property w.r.t. $R \in \mathfrak{R}$, abbreviated by $\operatorname{RP}(R)$, if for every sequence $\sigma=\left(a_{i}\right)_{i \in I}$ in $\mathfrak{S}$,

$$
\mu(\sigma)=b \Rightarrow\left\{\begin{array}{l}
\mu\left(\left|J_{\sigma}^{R}\right| \cdot b, a_{I \backslash J_{\sigma}^{R}}\right)=b \quad, \text { and } \\
\mu\left(a_{J_{\sigma}^{R}},\left|I \backslash J_{\sigma}^{R}\right| \cdot b\right)=b
\end{array}\right.
$$

(iii) $\mu$ is retractive w.r.t. $R \in \mathfrak{R}$, if for every $\sigma=\left(a_{i}\right)_{i \in I} \in \mathfrak{S}$ we have that $\mu\left(a_{I \backslash J_{\sigma}^{R}}\right)=$ $\mu(\sigma)$; 
(iv) $\mu$ is weakly retractive w.r.t. $R \in \mathfrak{R}$ if for every $\sigma=\left(a_{i}\right)_{i \in I} \in \mathfrak{S}$, we have that $|\mu(\sigma)| \geq\left|\mu\left(a_{I \backslash J_{\sigma}^{R}}\right)\right|$ with both of the same sign, i.e.,

$$
\mu(\sigma) \geq \mu\left(a_{I \backslash J_{\sigma}^{R}}\right) \geq 0 \quad \text { or } \quad \mu(\sigma) \leq \mu\left(a_{I \backslash J_{\sigma}^{R}}\right) \leq 0 .
$$

Lemma 3. For any $R \in \mathfrak{R}$, the symmetric maximum $\oslash_{R}$ is extremal, (weakly) retractive w.r.t. $R$, and satisfies $\operatorname{RP}(R)$.

Proof. Retractiveness and extremality are established in Lemma 1(i). As for $\operatorname{RP}(R)$, $b=\bigotimes_{R}(\sigma)=\bigotimes_{R}\left(a_{I \backslash J_{\sigma}^{R}}\right)$ by retractiveness, and $\bigotimes_{R}\left(a_{J_{\sigma}^{R}},\left|I \backslash J_{\sigma}^{R}\right| \cdot b\right)=b$ by decomposability for $K=I \backslash J_{\sigma}^{R}$. Now, since $a_{I \backslash J_{\sigma}^{R}}$ is associative and its extremal term is $b$, the sequence $\left(\left|J_{\sigma}^{R}\right| \cdot b, a_{I \backslash J_{\sigma}^{R}}\right)$ is still associative and $b$ is its extremal term.

Remark 1 . For any $R \in \mathfrak{R}$, the $\operatorname{RP}(R)$ property implies unanimity, i.e., $\mu(b, \ldots, b)=b$, for every $b \in \operatorname{Ran}\left(\mu\left(\mathfrak{S} \backslash \mathfrak{S}_{0}\right)\right)$, where $\operatorname{Ran}(\cdot)$ indicates the range of a function. Indeed, for $\sigma \in \mathfrak{S} \backslash \mathfrak{S}_{0}$ with $\mu(\sigma)=b, J_{\sigma}^{R}=\varnothing$.

\section{Characterizations}

We give several characterizations of weak $\mathbb{Z}$-means based on the symmetric maximum. The first one involves decomposability. We define $\operatorname{Ker}(\mu)=\left\{\sigma \in \mathfrak{S}^{*} \mid \mu(\sigma)=0\right\}$, where $\mathfrak{S}^{*}$ is the set of sequences in $\mathfrak{S}$ not containing 0 . Observe that by our convention, $\operatorname{Ker}(R)=\operatorname{Ker}\left(\otimes_{R}\right)$.

\subsection{Characterization by decomposability}

Theorem 3. Let $\mu: \tilde{C}^{*} \rightarrow \tilde{C}$ and let $R \in \mathfrak{R}$. Then $\mu=\bigotimes_{R}$ if and only if $\mu$ satisfies (C1), (C2), (C3) on $\tilde{C}^{2}$, is anonymous, weakly associative, decomposable on $J_{\sigma}^{R}$ for every sequence $\sigma$, and satisfies $\mu(\sigma)=0$ for every $\sigma \in \operatorname{Ker}(R)$ (i.e., $\operatorname{Ker}(R) \subseteq \operatorname{Ker}(\mu)$ ).

The following observation is useful in many subsequent proofs.

Observation 1. Let $\mu: \tilde{C}^{*} \rightarrow \tilde{C}$. If $\mu$ satisfies (C1), (C2), (C3), and weak associativity, then $\mu=\emptyset$ on $\mathfrak{S} \backslash \mathfrak{S}_{0}$.

Indeed, by Theorem 2 (C1), (C2), (C3) and weak associativity imply that $\mu$ coincides with $\oslash$ on $\tilde{C}^{2}$. It thus follows by weak associativity that $\mu(\sigma)=\emptyset(\sigma)$ for every associative sequence $\sigma$.

Proof. The "only if" part is obtained from previous results (in particular Lemma 2).

For the "if" part, by Observation $1, \mu=\mathbb{\emptyset}$ on $\mathfrak{S} \backslash \mathfrak{S}_{0}$.

Consider now sequences in $\mathfrak{S}_{0}$ and, for the sake of a contradiction, suppose that $\mu \neq \bigotimes_{R}$ on $\mathfrak{S}_{0}$. Take $\sigma=\left(a_{i}\right)_{i \in I}$ in $\mathfrak{S}_{0}$ such that $\mu(\sigma) \neq \bigotimes_{R}(\sigma)$ and $|\operatorname{Ran}(\sigma)|$ is minimum, where $\operatorname{Ran}(\sigma)$ is the range of $\sigma$ (i.e., the set of all integers in $\sigma$ ).

Suppose first that $|\operatorname{Ran}(\sigma)| \geq 3$. Note that $\left|\operatorname{Ran}\left(a_{J_{\sigma}^{R}}\right)\right| \geq 2$, so that $\mid \operatorname{Ran}\left(\left|J_{\sigma}^{R}\right|\right.$. $\left.\mu\left(a_{J_{\sigma}^{R}}\right), a_{I \backslash J_{\sigma}^{R}}\right)|<| \operatorname{Ran}(\sigma) \mid$. By definition of $\sigma$ and by applying decomposability on $J_{\sigma}^{R}$, it follows that

$$
\mu(\sigma)=\mu\left(a_{J_{\sigma}^{R}}, a_{I \backslash J_{\sigma}^{R}}\right)=\mu\left(\left|J_{\sigma}^{R}\right| \cdot \mu\left(a_{J_{\sigma}^{R}}\right), a_{I \backslash J_{\sigma}^{R}}\right)=\bigotimes_{R}\left(\left|J_{\sigma}^{R}\right| \cdot \mu\left(a_{J_{\sigma}^{R}}\right), a_{I \backslash J_{\sigma}^{R}}\right) .
$$


Now, by Lemma $1(\mathrm{iv}), a_{J_{\sigma}^{R}} \in \operatorname{Ker}(R) \subseteq \operatorname{Ker}(\mu)$, and since 0 is neutral for $\oslash_{R}$, we get

$$
\begin{aligned}
\bigotimes_{R}\left(\left|J_{\sigma}^{R}\right| \cdot \mu\left(a_{J_{\sigma}^{R}}\right), a_{I \backslash J_{\sigma}^{R}}\right) & =\bigotimes_{R}\left(\left|J_{\sigma}^{R}\right| \cdot 0, a_{I \backslash J_{\sigma}^{R}}\right) \\
& =\bigotimes_{R}\left(a_{I \backslash J_{\sigma}^{R}}\right)=\bigotimes_{R}(\sigma)
\end{aligned}
$$

where the last equality comes from Lemma 1(i). Combining the latter result with (8) yields $\mu(\sigma)=\bigotimes_{R}(\sigma)$, a contradiction.

Suppose now that $|\operatorname{Ran}(\sigma)|=2$ (note that a lower value is impossible). Then necessarily $\sigma$ is such that $\psi(\sigma)=\left(p_{1}, m_{1}\right)$ with $p_{1}>0, m_{1}>0$. Two cases arise. Suppose first that $a_{J_{\sigma}^{R}}=\sigma$. Then $\sigma \in \operatorname{Ker}(R)$, hence $\mu(\sigma)=0=\bigotimes_{R}(\sigma)$, a contradiction. Suppose on the contrary that $a_{J_{\sigma}^{R}} \neq \sigma$. Since $J_{\sigma}^{R} \in \operatorname{Ker}(R) \subseteq \operatorname{Ker}(\mu)$, it follows from decomposability for $J_{\sigma}^{R}$ that

$$
\mu(\sigma)=\mu\left(a_{I \backslash J_{\sigma}^{R}},\left|J_{\sigma}^{R}\right| \cdot \mu\left(a_{J_{\sigma}^{R}}\right)\right)=\mu\left(a_{I \backslash J_{\sigma}^{R}},\left|J_{\sigma}^{R}\right| \cdot 0\right) .
$$

Observe that by the assumption $|\operatorname{Ran}(\sigma)|=2$, we have necessarily $\left|\operatorname{Ran}\left(a_{I \backslash J_{\sigma}^{R}}\right)\right|=1$. Hence the sequence $\left(a_{I \backslash J_{\sigma}^{R}},\left|J_{\sigma}^{R}\right| \cdot 0\right)$ is associative, so that

$$
\mu\left(a_{I \backslash J_{\sigma}^{R}},\left|J_{\sigma}^{R}\right| \cdot 0\right)=\bigotimes_{R}\left(a_{I \backslash J_{\sigma}^{R}},\left|J_{\sigma}^{R}\right| \cdot 0\right)=\bigotimes_{R}\left(a_{I \backslash J_{\sigma}^{R}}\right)=\bigotimes_{R}(\sigma)
$$

Combining this with $(9)$, we find $\mu(\sigma)=\bigotimes_{R}(\sigma)$, again a contradiction.

Remark 2. If $R=\langle\cdot\rangle_{-}^{+}$, then decomposability on $J_{\sigma}^{R}$ is trivially satisfied and therefore is not needed in the axiomatization. Indeed, $J_{\sigma}^{(\cdot\rangle_{-}^{+}}=I$ for any $\sigma=\left(a_{i}\right)_{i \in I} \in \mathfrak{S}_{0}$, and letting $\mu(\sigma)=b$, we have $\mu(b, \ldots, b)=\otimes(b, \ldots, b)=b$ by Observation 1 .

\subsection{Characterization by the replacement property and others}

We introduce further characterizations based on the properties in Definition 1. First, we observe that the kernels of $\mu$ and $R$ are somehow related.

Lemma 4. Suppose that $\mu: \tilde{C}^{*} \rightarrow \tilde{C}$ satisfies (C1), (C2), (C3) on $\tilde{C}^{2}$ and weak associativity. If $\mu$ verifies $\operatorname{RP}(R)$ for some $R \in \mathfrak{R}$, then $\operatorname{Ker}(\mu) \subseteq \operatorname{Ker}(R)$. If in addition $\left|\oslash_{R}(\sigma)\right| \geq|\mu(\sigma)|$ for every $\sigma \in \mathfrak{S}$, denoted by $\left|\bigotimes_{R}\right| \geq|\mu|$, then $\operatorname{Ker}(R)=\operatorname{Ker}(\mu)$.

Proof. Note that by $(\mathrm{C} 2) \operatorname{Ker}(\mu) \neq \varnothing$. For the sake of a contradiction, suppose that there exists $\sigma=\left(a_{i}\right)_{i \in I} \in \operatorname{Ker}(\mu) \backslash \operatorname{Ker}(R)$, i.e., $\mu(\sigma)=0$ and $\emptyset_{R}(\sigma) \neq 0$. As before, take such a $\sigma$ with minimum $|\operatorname{Ran}(\sigma)|$. As $\mu$ satisfies $(\mathrm{C} 1)$ and $(\mathrm{C} 2)$, we may assume that $|\operatorname{Ran}(\sigma)| \geq 2$.

The case $|\operatorname{Ran}(\sigma)|=2$ is dealt with as above. So suppose that $|\operatorname{Ran}(\sigma)| \geq 3$. Observe that $J_{\sigma}^{R}=\varnothing$ is impossible. Indeed, this would mean that $\sigma \in \mathfrak{S}^{*} \backslash \mathfrak{S}_{0}$, and by Observation $1,0=\mu(\sigma)=\otimes(\sigma)$, a contradiction. Hence, $J_{\sigma}^{R} \neq \varnothing$. Then

$$
\left|\operatorname{Ran}\left(\left|J_{\sigma}^{R}\right| \cdot 0, a_{I \backslash J_{\sigma}^{R}}\right)\right|<|\operatorname{Ran}(\sigma)| .
$$

By $\operatorname{RP}(R)$,

$$
\mu\left(a_{J_{\sigma}^{R}},\left|I \backslash J_{\sigma}^{R}\right| \cdot 0\right)=0=\mu\left(\left|J_{\sigma}^{R}\right| \cdot 0, a_{I \backslash J_{\sigma}^{R}}\right) .
$$

By the minimality assumption on $\sigma$, it then follows from (10) that

$$
0=\mu\left(\left|J_{\sigma}^{R}\right| \cdot 0, a_{I \backslash J_{\sigma}^{R}}\right)=\bigotimes_{R}\left(\left|J_{\sigma}^{R}\right| \cdot 0, a_{I \backslash J_{\sigma}^{R}}\right) .
$$


By Lemma 1 (i) and the fact that 0 is neutral in $\otimes_{R}$, we then have that

$$
0=\bigotimes_{R}\left(\left|J_{\sigma}^{R}\right| \cdot 0, a_{I \backslash J_{\sigma}^{R}}\right)=\bigotimes_{R}\left(a_{I \backslash J_{\sigma}^{R}}\right)=\bigotimes_{R}(\sigma),
$$

which contradicts the choice of $\sigma$. Hence there is no $\sigma \in \operatorname{Ker}(\mu) \backslash \operatorname{Ker}(R)$, and thus $\operatorname{Ker}(\mu) \subseteq \operatorname{Ker}(R)$.

The last assertion is obvious.

Remark 3. If $\mu: \tilde{C}^{*} \rightarrow \tilde{C}$ satisfies (C1), (C2), (C3), is weakly associative and weakly retractive w.r.t. $R$, then by Observation $1,\left|\oslash_{R}\right| \leq|\mu|$.

Proposition 1. Let $\mu: \tilde{C}^{*} \rightarrow \tilde{C}$ be a function that satisfies (C1), (C2), (C3) on $\tilde{C}^{2}$. Then the following assertions are equivalent:

(i) $\mu=\bigotimes_{R}$;

(ii) $\mu$ is extremal w.r.t. $R \in \mathfrak{R}$;

(iii) $\mu$ is weakly associative and it is retractive w.r.t. $R$;

(iv) $\mu$ is weakly associative, weakly retractive, and $\left|\oslash_{R}\right| \geq|\mu|$.

Proof. By Lemma 1(i), (i) is equivalent to (ii) and (i) implies (iii). Also, if $\mu$ satisfies (C1), (C2), (C3) on $\tilde{C}^{2}$ and it is weakly associative and retractive w.r.t. $R$, then

$$
\mu(\sigma)=\mu\left(a_{I \backslash J_{\sigma}^{R}}\right)=\left(\min _{i \in I \backslash J_{\sigma}^{R}} a_{i}\right) \otimes\left(\max _{i \in I \backslash J_{\sigma}^{R}} a_{i}\right),
$$

that is, $\mu$ is extremal w.r.t. $R$, and hence (iii) implies (ii).

Also, by Lemma 3 we have that (i) implies (iv). To show that the converse implication also holds, note that by Remark 3 and $\left|\oslash_{R}\right| \geq|\mu|$ we get $\left|\oslash_{R}\right|=|\mu|$ and, by weak retractiveness and Observation 1 , it follows that $\emptyset_{R}=\mu$.

Lemma 5. Suppose that $\mu: \tilde{C}^{*} \rightarrow \tilde{C}$ satisfies (C1), (C2), (C3) on $\tilde{C}^{2}$, and that $\mu$ is weakly associative, anonymous, and satisfies $\operatorname{RP}(R)$ and $\left|\oslash_{R}\right| \geq|\mu|$ with both of the same sign (i.e., $\mathbb{\bigotimes}_{R}(\sigma) \geq 0$ if and only if $\mu(\sigma) \geq 0$ ), for some $R \in \mathfrak{R}$. Then $\mu=\bigotimes_{R}$.

Proof. First we show that $\left|\bigotimes_{R}\right|=|\mu|$. To this end, note that from (C1), (C2), (C3) and weak associativity it follows by Observation 1 that for any sequence $\sigma=\left(a_{i}\right)_{i \in I} \in \mathfrak{S}_{0}$,

$$
\mu\left(a_{I \backslash J_{\sigma}^{R}}\right)=\bigotimes_{R}\left(a_{I \backslash J_{\sigma}^{R}}\right)=\bigotimes_{R}(\sigma)
$$

and, since $\left|\oslash_{R}\right| \geq|\mu|$, we cannot have $|\mu(\sigma)|>\left|\mu\left(a_{I \backslash J_{\sigma}^{R}}\right)\right|$. Hence, $|\mu(\sigma)| \leq\left|\mu\left(a_{I \backslash J_{\sigma}^{R}}\right)\right|$.

Now, suppose that $|\mu(\sigma)|<\left|\mu\left(a_{I \backslash J_{\sigma}^{R}}\right)\right|$. By (11) and Lemma 1(i),

$$
\left|\mu\left(a_{I \backslash J_{\sigma}^{R}}\right)\right|=\left|\oslash\left(a_{I \backslash J_{\sigma}^{R}}\right)\right|=\left|\bigotimes_{R}\left(a_{I \backslash J_{\sigma}^{R}}\right)\right|=\left|\bigotimes_{R}(\sigma)\right|=\max _{i \in I \backslash J_{\sigma}^{R}}\left|a_{i}\right| .
$$

Let $b=\mu(\sigma)$. Since $\mu$ and $\oslash_{R}$ have the same sign and $\mu$ is odd, we may assume that $b \geq 0$. By assumption and (12), we have that

$$
b<\max _{i \in I \backslash J_{\sigma}^{R}}\left|a_{i}\right|=\left|\bigotimes_{R}(\sigma)\right| .
$$


$\operatorname{By} \operatorname{RP}(R)$, we have that $\mu(\sigma)=\mu\left(\left|J_{\sigma}^{R}\right| \cdot b, a_{I \backslash J_{\sigma}^{R}}\right)$, and since $\left(\left|J_{\sigma}^{R}\right| \cdot b, a_{I \backslash J_{\sigma}^{R}}\right)$ is associative (by (13)),

$$
\mu(\sigma)=\mu\left(\left|J_{\sigma}^{R}\right| \cdot b, a_{I \backslash J_{\sigma}^{R}}\right)=\bigotimes_{R}\left(\left|J_{\sigma}^{R}\right| \cdot b, a_{I \backslash J_{\sigma}^{R}}\right) .
$$

However, $\otimes_{R}$ is extremal w.r.t. $R$ and from the fact that $0 \leq b<\max _{i \in I \backslash J_{\sigma}^{R}}\left|a_{i}\right|$ it follows from Lemma 1(i) that

$$
\bigotimes_{R}\left(\left|J_{\sigma}^{R}\right| \cdot b, a_{I \backslash J_{\sigma}^{R}}\right)=\bigotimes_{R}\left(a_{I \backslash J_{\sigma}^{R}}\right)=\bigotimes_{R}(\sigma),
$$

and thus by (14)

$$
\mu(\sigma)=\mu\left(\left|J_{\sigma}^{R}\right| \cdot b, a_{I \backslash J_{\sigma}^{R}}\right)=\bigotimes_{R}\left(\left|J_{\sigma}^{R}\right| \cdot b, a_{I \backslash J_{\sigma}^{R}}\right)=\bigotimes_{R}(\sigma) .
$$

Hence, $\bigotimes_{R}(\sigma)=b$, which contradicts (13), and thus $|\mu(\sigma)|=\left|\mu\left(a_{I \backslash J_{\sigma}^{R}}\right)\right|=\left|\bigotimes_{R}(\sigma)\right|$, as desired. Since $\otimes_{R}$ and $\mu$ have the same sign, it then follows that $\emptyset_{R}=\mu$.

Remark 4. As for the previous characterization, the rule $R=\langle\cdot\rangle_{-}^{+}$is particular, because the property $\left|\oslash_{R}\right| \geq|\mu|$ with the same sign is enough to characterize it, without the help of $R P(R)$.

We can now state further characterizations as mentioned earlier.

Theorem 4. Let $\mu: \tilde{C}^{*} \rightarrow \tilde{C}$ be an anonymous and weakly associative function that satisfies (C1), (C2), (C3) on $\tilde{C}^{2}$. For every $R \in \mathfrak{R}$, the following assertions are equivalent.

(i) $\mu=\bigotimes_{R}$.

(ii) $\mu$ decomposable on $J_{\sigma}^{R}$ for every sequence $\sigma$, and $\operatorname{Ker}(R) \subseteq \operatorname{Ker}(\mu)$.

(iii) $\mu$ is extremal w.r.t. $R$.

(iv) $\mu$ is retractive w.r.t. $R$.

(v) $\mu$ is weakly retractive and $\left|\oslash_{R}\right| \geq|\mu|$.

(vi) $\mu$ verifies $\operatorname{RP}(R)$ and $\left|\oslash_{R}\right| \geq|\mu|$ both with the same sign.

Proof. By Theorem 3 and Proposition 1 we have that (i) to (v) are all equivalent. By Lemma 5 we also have that (vi) implies (i), and by Lemma 3 it follows that (i) implies (vi).

\subsection{Independence of the axioms}

As a preliminary remark, we must distinguish the roles of the different axioms introduced. Axioms (C1), (C2), (C3) pertain only to the case of a binary operator and are clearly independent. Weak associativity permits to extend to associative sequences, and by Observation 1, the operator $\otimes$ is obtained. Hence, the four axioms are independent.

The remaining axioms only serve to determine what happens for nonassociative sequences. Anonymity permits to work on ordered sequences and is clearly independent of the other remaining axioms.

Therefore, our task consists in proving that, for any rule $R$ (except for $R=\langle\cdot\rangle_{-}^{+}$, see Remarks 2 and 4) 
(i) in Theorem 3, decomposability on $J_{\sigma}^{R}$ and $\operatorname{Ker}(R) \subseteq \operatorname{Ker}(\mu)$ are independent, and

(ii) in Lemma $5, \operatorname{RP}(R)$ and $\left|\bigotimes_{R}\right| \geq|\mu|$ with the same sign, are also independent.

We begin with Theorem 3 and suppose that $\mu$ satisfies (C1), (C2), (C3), anonymity, and weak associativity. Consider any rule $R \neq\langle\cdot\rangle_{-}^{+}$in $\mathfrak{R}$. Clearly, we only have to consider nonassociative sequences $\sigma=\left(a_{i}\right)_{i \in I}$. Let $\tilde{C}=\mathbb{Z}$.

(i) Define $\mu(\sigma)$ as $\min \left(a_{I \backslash J_{\sigma}^{R}}\right)$ for any nonassociative $\sigma$ s.t. $I \backslash J_{\sigma}^{R} \neq \varnothing$, and 0 otherwise. Then $\mu$ satisfies $\operatorname{Ker}(R) \subseteq \operatorname{Ker}(\mu)$ but not decomposability. Indeed, by Lemma 1(iv)

$$
\mu\left(a_{J_{\sigma}^{R}}\right)=0 \text { and } \mu\left(\left|J_{\sigma}^{R}\right| \cdot 0, a_{I \backslash J_{\sigma}^{R}}\right)=\otimes\left(\left|J_{\sigma}^{R}\right| \cdot 0, a_{I \backslash J_{\sigma}^{R}}\right)=\bigotimes\left(a_{I \backslash J_{\sigma}^{R}}\right) \neq \min \left(a_{I \backslash J_{\sigma}^{R}}\right)
$$

in general. For instance, take $\sigma=(3,-3,2,1)$.

(ii) Define $\mu\left(a_{J_{\sigma}^{R}}\right)=\alpha \in C, \alpha>0$, for any $\sigma$, except if $a_{J_{\sigma}^{R}}=(a,-a)$, in which case $\mu\left(a_{J_{\sigma}^{R}}\right)=0$, so that $(\mathrm{C} 2)$ is satisfied. Then, $\operatorname{Ker}(R) \subseteq \operatorname{Ker}(\mu)$ is not satisfied. We claim that, assuming that $\mu$ is decomposable on $J_{\sigma}^{R}, \mu$ is well defined on $\mathfrak{S}_{0}$ (note that by $(\mathrm{C} 2)$, we already have $\mu(a,-a)=0$ for every $a$.). Indeed, by decomposability, $\mu(\sigma)=\mu\left(\sigma_{J_{\sigma}^{R}}, \sigma_{I \backslash J_{\sigma}^{R}}\right)=\mu\left(\left|J_{\sigma}^{R}\right| \cdot \alpha, \sigma_{I \backslash J_{\sigma}^{R}}\right)$ or $\mu\left(\left|J_{\sigma}^{R}\right| \cdot 0, \sigma_{I \backslash J_{\sigma}^{R}}\right)$. In the latter case, $\left(\left|J_{\sigma}^{R}\right| \cdot 0, \sigma_{I \backslash J_{\sigma}^{R}}\right)=: \sigma^{\prime}$ is associative, so that $\mu(\sigma)=\emptyset\left(\sigma^{\prime}\right)$. In the former case, if $-\alpha$ is not extremal in $\sigma_{I \backslash J_{\sigma}^{R}}$, the sequence $\sigma^{\prime}=\left(\left|J_{\sigma}^{R}\right| \cdot \alpha, \sigma_{I \backslash J_{\sigma}^{R}}\right)$ is associative, and therefore $\mu(\sigma)=\otimes\left(\sigma^{\prime}\right)$. Otherwise, $\sigma_{I \backslash J_{\sigma}^{R}}$ contains $-\alpha$ as extremal term and possibly other terms smaller in magnitude. By repeatedly applying decomposability, say $k \geq 1$ times, we can delete the occurrence of $-\alpha$ in the subsequence that remains undeleted, and thus we arrive at a sequence $\sigma^{(k)}=\left(\left|J_{\sigma^{(k-1)}}^{R}\right| \cdot \alpha, \sigma_{I \backslash J_{\sigma^{(k-1)}}^{R}}^{(k-1)}\right)$ that is associative. To illustrate this fact, suppose that $\sigma=(2 \cdot 3,7 \cdot(-3)), \stackrel{\sigma}{R}=\langle\cdot\rangle_{0}=\rho_{3}^{*}$, and that $\mu(n \cdot 3, n \cdot(-3))=\alpha=3$, for $n \geq 2$. Then the first application of decomposability produces $\sigma^{\prime}=\left(\left|J_{\sigma}^{R}\right| \cdot \alpha, \sigma_{I \backslash J_{\sigma}^{R}}\right)=(4 \cdot 3,5 \cdot(-3))$ and thus $\sigma_{I \backslash J_{\sigma^{\prime}}^{R}}^{\prime}=(-3)$. By reapplying decomposability, we obtain $\sigma^{(2)}=\left(\left|J_{\sigma^{\prime}}^{R}\right| \cdot \alpha, \sigma_{I \backslash J_{\sigma^{\prime}}^{R}}^{\prime}\right)=(8 \cdot 3,1 \cdot(-3))$ and in this case we have $\sigma_{I \backslash J_{\sigma^{(2)}}}^{(2)}=(7 \cdot 3)$. By applying decomposability for a third time, we get $\sigma^{(3)}=\left(\left|J_{\sigma^{(2)}}^{R}\right| \cdot \alpha, \sigma_{I \backslash J_{\sigma^{(2)}}^{R}}\right)=(9 \cdot 3)$, which is now associative, and from which it follows that

$$
\mu(\sigma)=\mu\left(\sigma^{\prime}\right)=\mu\left(\sigma^{(2)}\right)=\mu\left(\sigma^{(3)}\right)=\emptyset\left(\sigma^{(3)}\right)=3 .
$$

In general, if $k \geq 1$ applications of decomposability suffice to delete the occurrences of $-\alpha$ in $\sigma_{I \backslash J_{\sigma}^{R}}$ (i.e., $\sigma^{(k)}$ is associative), then

$$
\mu(\sigma)=\mu\left(\sigma^{\prime}\right)=\cdots=\mu\left(\sigma^{(k)}\right)=\emptyset\left(\sigma^{(k)}\right)=\alpha .
$$

Let us now turn to Lemma 5 and suppose as before that $R \neq\langle\cdot\rangle_{-}^{+}$.

(i) Define $\mu$ by $\mu(\sigma)=0$ for every $\sigma \in \operatorname{Ker}(R)$, otherwise, $\mu(\sigma)$ is the value in $\sigma_{I \backslash J_{\sigma}^{R}}$ which has smallest absolute value, with the sign of the extremal value of $\sigma_{I \backslash J_{\sigma}^{R}}$ (Example: $\mu(3,-3,-2,1)=-1$ with $\left.R=\langle\cdot\rangle_{0}\right)$. Then $\mu$ satisfies $\left|\bigotimes_{R}\right| \geq|\mu|$ with the same sign. However, $R P(R)$ is not satisfied, whenever $\sigma$ is such that $I \backslash J_{\sigma}^{R} \neq \varnothing$, with at least two values of different magnitude. 
(ii) Consider $\tilde{C}=\mathbb{Z}$ and define $\mu$ by $\mu(\sigma)=0$ for every $\sigma \in \operatorname{Ker}(R)$, otherwise

$$
\mu(\sigma)=\operatorname{sgn}\left(\text { extremal value of } \sigma_{I \backslash J_{\sigma}^{R}}\right) \cdot\left(\min _{i \in J_{\sigma}^{R}}\left(\left|a_{i}\right|\right)-1\right) .
$$

For example, $\mu(4,-4,3,-3,-1)=-2$ with $R=\langle\cdot\rangle_{0}$. As the example shows, the property $\left|\emptyset_{R}\right| \geq|\mu|$ fails. We claim that $\mu$ satisfies $R P(R)$. Indeed, for any $\sigma \notin \operatorname{Ker}(R)$, letting $\mu(\sigma)=b$, we have $\mu\left(\left|J_{\sigma}^{R}\right| \cdot b, \sigma_{I \backslash J_{\sigma}^{R}}\right)=b$ because $\left(\left|J_{\sigma}^{R}\right| \cdot b, \sigma_{I \backslash J_{\sigma}^{R}}\right)$ is associative with extremal value $b$, and $\mu\left(\sigma_{J_{\sigma}^{R}},\left|I \backslash J_{\sigma}^{R}\right| \cdot b\right)=b$, since for $\sigma^{\prime}=$ $\left(\sigma_{J_{\sigma}^{R}},\left|I \backslash J_{\sigma}^{R}\right| \cdot b\right)$ we have $J_{\sigma^{\prime}}^{R}=J_{\sigma}^{R}$.

\section{References}

[1] C. D. Bennett, W. C. Holland, and G. J. Székely. Integer valued means. Aequat. Math., 88:137-149, 2014.

[2] M. Couceiro and M. Grabisch. On the poset of computation rules for nonassociative calculus. Order, 30:269-288, 2013. DOI 10.1007/s11083-011-9243-z.

[3] M. Grabisch. The Möbius function on symmetric ordered structures and its application to capacities on finite sets. Discrete Mathematics, 287(1-3):17-34, 2004.

[4] M. Grabisch, J.-L. Marichal, R. Mesiar, and E. Pap. Aggregation functions. Number 127 in Encyclopedia of Mathematics and its Applications. Cambridge University Press, 2009.

[5] A. Kolmogoroff. Sur la notion de moyenne. Atti delle Reale Accademia Nazionale dei Lincei Mem. Cl. Sci. Fis. Mat. Natur. Sez., 12:323-343, 1930.

[6] J.-L. Marichal and B. Teheux. Barycentrically associative and preassociative functions. Acta Math. Hungar., 145:468-488, 2015.

[7] M. Nagumo. Über eine Klasse der Mittelwerte. Japanese Journal of Mathematics, 7:71-79, 1930. 